\title{
Immunology and Diabetes Workshop: Report on the Third International (Stage 3) Workshop on the Standardisation of Cytoplasmic Islet Cell Antibodies
}

\author{
Held in New York, New York, October 1987 \\ C. Boitard, E. Bonifacio, G. F. Bottazzo, H. Gleichmann and J.Molenaar \\ Hôpital Necker, INSERM U25, Paris, France
}

Identification of individuals at risk for developing Type 1 (insulindependent) diabetes mellitus, diagnosis of Type 1 diabetes in cases of unusual clinical presentation, and monitoring of Type 1 patients undergoing various controlled therapeutic trials are major areas in which islet cell antibody (ICA) determination has been employed as a key immunological marker in diabetes investigation. Since ICA were first described in 1974, numerous methodological modifications of the original indirect immunofluorescence (IFL) assay and new approaches to anti-islet antibody detection have been proposed. The need to compare the various tests was the basis for the First International (Stage 1) Workshop on ICA standardisation in Monaco, 1985. Results then obtained with coded samples indicated that availability of reference sera should allow standard curves to be established and used by individual laboratories to express ICA in arbitary, but common, units. A reference standard - JDF (Juvenile Diabetes Fondation) standard - was proposed and tested in the Stage 2 Workshop held in Perth, Australia, 1987, which examined the use of standard curves to improve intra-assay and inter-assay precision and concordance between laboratories.

The Stage 3 Workshop was proposed so as to assess precision of individual laboratories, the specificity of ICA, the improvement of interlaboratory concordance by using the JDF standard and expressing results in common arbitrary units; and to evaluate the role of intra-laboratory precision on inter-laboratory concordance.

Forty-three coded freeze-dried serum samples were prepared and distributed to participants along with a disclosed $2.0 \mathrm{ml}$ freeze-dried aliquot of the JDF standard serum. The set of coded samples included a) 10 unknown blood bank donor sera and 2 selected blood bank donor sera detected as ICA positive by some of the participating laboratories b) 15 samples in duplicate being aliquots of [i] the JDF standard serum undiluted, $1: 2,1: 4,1: 8$, and $1: 16$ in negative serum, [ii] a second standard made from a pool of sera collected from $7-15$ year-old recent-onset Type 1 diabetic children undiluted, $1: 2,1: 4,1: 8$ and $1: 16$ in negative serum and [iii] a third standard made from a pool of 10 high titre ICA positive sera again diluted undiluted, $1: 2,1: 4,1: 8,1: 16$ in negative serum. The negative serum diluent was also included.

Results were available from 43 assays performed in 40 laboratories. Four laboratories did not detect ICA in any of the samples and these were not included in the remainder of the analysis. Of the remaining 39 assays, 23 assays employed conventional IFL, 10 incorporated extended incubation of sera, 3 used two color immunofluorescence, 2 protein $A$ binding and 1 biotin-avidin labelling. Thirtyfive laboratories used human substrates and 4 animal (monkey, baboon, rat) substrates.

The 15 duplicate pairs were analysed to determine intra-laboratory precision as indicated by standard deviations of duplicate geometric titre differences and the ability to discriminate the standard serum dilution intervals. There were substantial differences in intralaboratory precision of individual laboratories. By ranking their

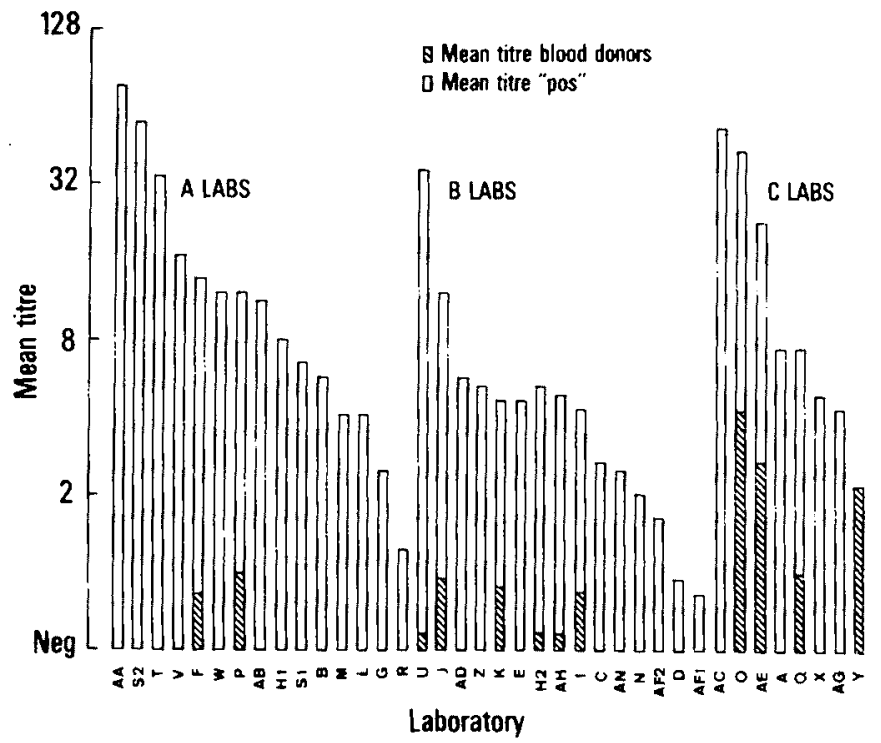

Fig. 1. Stage III ICA Workshop: mean signal vs noise. Mean ICA titres of the 30 camples of the 3 standard sera dilutions ("positive") and mean titre of blood bank donor sera ( $y$ axis) in each participating laboratory ( $x$ axis) ranked depending on precision $(A>B>C)$

precision score, laboratories were assigned to arbitrary groups $[A][B]$ [C]. Ranking assignment of precision correlated neither with the method or the substrate used but clearly correlated with the number of previous participations in Stage 1 and 2. Laboratories which had participated in all 3 stages of the workshop as a whole had better precision than those laboratories which had joined the standardisation programme at stage 2 or 3 .

The qualitative analysis of the results obtained with the blood bank donor sample showed a concordance of $81.89 \%$ between participating laboratories with regard to the absence of ICA. In 25 out of 39 assays, all 10 random blood bank donor samples were ICA negative. As shown in Figures 1 and 2, disease specificity was clearly, although not exclusively, dependent on intra-laboratory precision. Very large differences in ICA titres and analytical sensitivity between laboratories is also shown in Figure 1.

Conversion of titres into units using the JDF standard dilution curve increased concordance amongst laboratories with highest precision. Laboratories with poor precision also had poor inter-laboratory concordance and correlated least with the concensus. Inter-laboratory concordance was thus dependent on intra-laboratory precision (Fig.2). An independent analysis based on accuracy was 


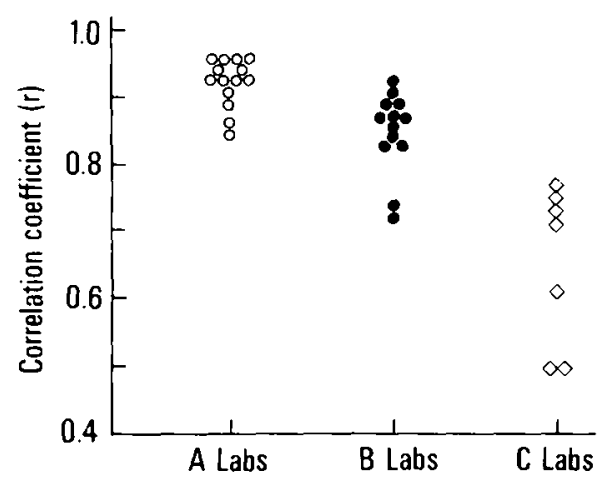

Fig. 2. Inter-laboratory concordance. Correlation coefficient of individual laboratory data expressed as common (JDF) units from their JDF dilution curve against the mean consensus value from all participating laboratories. Laboratories are ranked as in Figure 1 performed on 28 assays which allowed construction of acceptable standard curves. This analysis reached similar conclusions.

In conclusion, the available standards allowed evaluation of precision and demonstrated that good precision can be achieved. This is a prerequisite for future evaluation of predictive values of ICA determination in different clinical situations. Recommendations will be made based on the use of standard sera for future scientific works using ICA detection. Available standards will be used to calibrate proficiency sera which will be available on a regular basis to laboratories using islet cell antibody assays.

For further information about future ICA standardisation, contact Dr. Christian Boitard, secretary of the IDW committee.

Hôpital Necker

INSERM U 25

161 rue de Sèvres

F-75743 Paris Cedex 15

France 